\title{
Les repas culpabilisés de la poésie grecque contemporaine
}

The Guilty Meals in Contemporary Greek Poetry

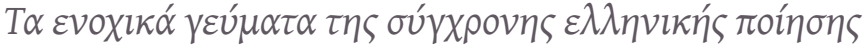

\section{Théodouli Alexiadou}

\section{CpenEdition}

\section{Journals}

Édition électronique

URL : https://journals.openedition.org/ceb/7258

DOI : $10.4000 /$ ceb.7258

ISSN : 2261-4184

\section{Éditeur}

INALCO

Édition imprimée

ISBN : 978-2-85831-230-6

ISSN : 0290-7402

\section{Référence électronique}

Théodouli Alexiadou, "Les repas culpabilisés de la poésie grecque contemporaine », Cahiers

balkaniques [En ligne], Hors-série | 2016, mis en ligne le 23 mars 2017, consulté le 06 juillet 2021. URL : http://journals.openedition.org/ceb/7258 ; DOl : https://doi.org/10.4000/ceb.7258

Ce document a été généré automatiquement le 6 juillet 2021

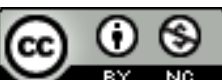

Cahiers balkaniques est mis à disposition selon les termes de la Licence Creative Commons Attribution - Pas d'Utilisation Commerciale 4.0 International. 


\title{
Les repas culpabilisés de la poésie grecque contemporaine
}

\author{
The Guilty Meals in Contemporary Greek Poetry

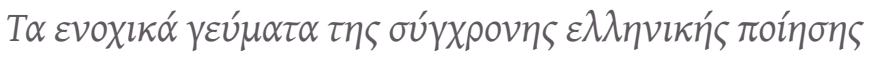

\section{Théodouli Alexiadou}

1 Pour Épicure, la satisfaction de l'estomac est la recherche de l'«aponie » du corps, c'est-à-dire la satisfaction de ses besoins, qui a pour effet l'« ataraxie »1' de l'âme, donc le bonheur ${ }^{2}$. La philosophie épicurienne considère que le corps joue un rôle déterminant dans l'élaboration de la morale et soutient que « le principe et la racine de tout bien, c'est le plaisir du ventre " tandis que « l'absence de faim est un élément d'eudémonie " ${ }^{3}$. Mais si l'absence de faim mène à l'« aponie » et à l'" ataraxie», la nourriture en tant que condition à la survie pose les limites et les contraintes de l'existence physique. Emmanuel Lévinas résume, dans la phrase qui suit, ces états complémentaires, le besoin et le plaisir :

Il n'est peut-être pas juste de dire que nous vivons pour manger, mais il n'est pas plus juste de dire que nous mangeons pour vivre ${ }^{4}$.

2 Dans la poésie grecque moderne, l'importance de la nourriture en tant que signifié et symbole altère sa dimension à la fois «morale » et sensuelle. L'apaisement que procure la survie, le plaisir, la joie du vivre ensemble à travers un repas partagé, se rencontrent rarement et même si cela arrive, on peut difficilement éviter les co-déclarations culpabilisées. La relation de la nourriture avec l'individualisme et avec la mort crée un cadre " anorexique ", avec tout ce que certaines exceptions peuvent engendrer comme brèches. Le décor serein que nous trouvons dans le Dîner d'Anghélos Sikélianos, au début du Xx $x^{e}$ siècle, s'est depuis longtemps évanoui ${ }^{5}$ :

\begin{tabular}{|c|c|}
\hline 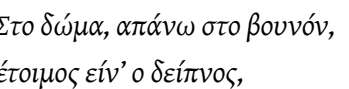 & $\begin{array}{l}\text { Dans la cabane, là-haut, sur } \\
\text { la montagne, le dîner est prêt, }\end{array}$ \\
\hline
\end{tabular}




\begin{tabular}{|c|c|}
\hline 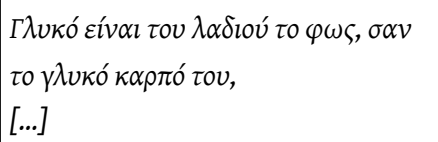 & $\begin{array}{l}\text { Douce est la lueur de l'huile, } \\
\text { comme doux est son fruit } \\
{[\ldots]}\end{array}$ \\
\hline 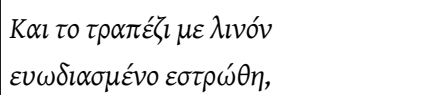 & $\begin{array}{l}\text { Et sur la table, recouverte de } \\
\text { lin qui embaume }\end{array}$ \\
\hline 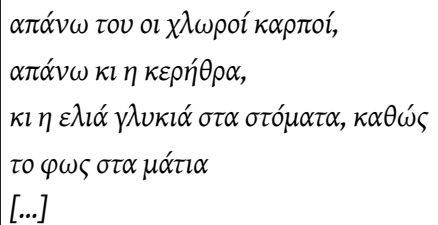 & $\begin{array}{l}\text { Les fruits frais } \\
\text { et la cire } \\
\text { Et l'olive douce à la bouche, } \\
\text { comme la lumière dans les yeux } \\
{[\ldots . .}\end{array}$ \\
\hline
\end{tabular}

\section{De la carnivorité}

Dans les repas poétiques contemporains, l'« ataraxie » fait place à l'angoisse et le plaisir est contaminé par la peur et la culpabilité. Le cas le plus significatif de tous est celui de Yannis Varveris: dans sa poésie, les symboles alimentaires entrent dans le cadre oppressant de l'insolite et de la non consommation. Le repas familial par excellence, celui du dimanche midi, associé au cycle de la vie et clôturant la semaine en réunissant autour de la table les personnes aimées ${ }^{6}$, devient une sorte de Cène où la présence de la mort est mise en évidence et la notion de trahison soulignée.

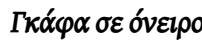

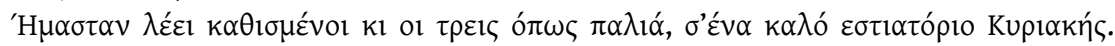

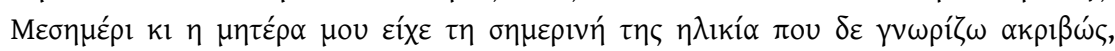

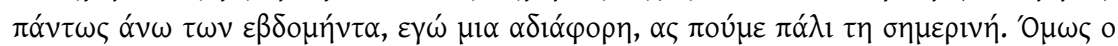

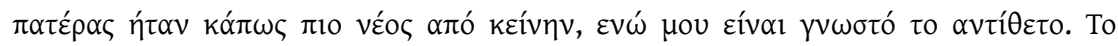

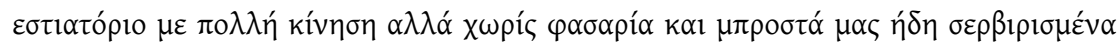

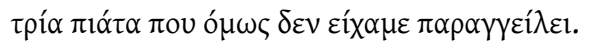

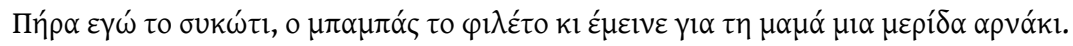

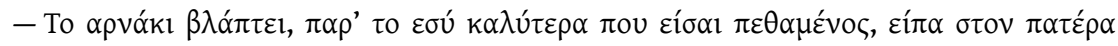
$\mu$ ov.

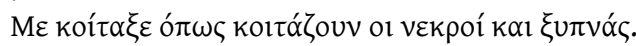

\section{Une gaffe dans un rêve ${ }^{7}$}

On était assis tous les trois comme autrefois, dans un bon restaurant, un dimanche à midi et ma mère avait l'âge qu'elle a aujourd'hui et que je ne connais pas exactement, plus de soixante-dix ans en tout cas ; moi, un âge quelconque... disons... là aussi... celui que j'ai aujourd'hui. Sauf que père était un peu plus jeune qu'elle, alors que je sais que c'est le contraire. Dans le restaurant, une grande affluence mais pas de vacarme, et devant nous, déjà, trois plats qu'on n'avait pourtant pas commandés.

Moi j'ai pris du foie, papa, le morceau de filet et l'assiette d'agneau est restée pour maman.

- L'agneau, c'est mauvais pour la santé, prends-le toi plutôt puisque tu es mort, j'ai dit à papa. Il m'a regardé comme regardent les morts... et après on se réveille !

Les conditions d'une absence de réciprocité sont posées par le fait que le repas se déroule dans un restaurant et que les personnes ne partagent pas les mêmes saveurs, comme cela se passerait lors d'un repas à la maison. Le partage se transforme en choix, et les trois plats différents et déjà servis seront la cause de la trahison qui va suivre. Les convives mâles sont les premiers à choisir : le narrateur, du foie, et le père, un morceau 
de filet. La mère prend ce qui reste, de l'agneau. Mais que signifient ces aliments et pourquoi le narrateur les redistribue-t-il, commettant ainsi la « gaffe » du titre?

5 Tout d'abord, la viande et les différents morceaux de la bête ne sont, en aucun cas, des éléments neutres. Pour la culture occidentale, la couleur rouge de la viande détermine sa force. La consommation de viande symbolise l'appropriation de l'énergie de l'animal et, surtout, du sang qu'il contient. De surcroît, plus évidente est la provenance du morceau qui est consommé, moins il est traité par la cuisson ou le découpage, plus il est considéré comme étant «vivant » et moins «masqué » et plus son consommateur est symboliquement culpabilisé 8 .

6 Eu égard à ce qui précède, on prend conscience que le foie choisi par le narrateur-fils comporte la plus grande quantité de sang et donc, la plus grande force, tandis qu'il renvoie directement à sa provenance initiale, sous-entendant ainsi la culpabilisation du mangeur, puisque c'est le morceau le plus «vivant ». Dans la poésie de Varveris tout particulièrement, si l'on prend en compte une donnée biographique, celle du poète qui était souvent soumis à des dialyses, l'importance symbolique du sang est énorme et ne fait pas seulement allusion à la force qui est transférée en celui qui l'« intègre » mais à la survivance elle-même.

7 Dans ce rêve, aucun des personnages ne consomme de nourriture, ni ne semble avoir faim, pas plus qu'il ne semble à la recherche du plaisir. Passifs, ils acceptent avec fatalité les plats qui ont été choisis pour eux et le sujet poétique se charge de redistribuer la nourriture en prenant pour critère ses caractéristiques vitales ou mortifères. Le regard du père confirme la culpabilité du narrateur qui assume la responsabilité du sacrifice. Les trois plats d'un repas de famille dominical, sans même qu'ils soient consommés, déterminent en termes de vie et de mort le destin des personnages. Le mangeur s'approprie les qualités symboliques du repas et le symbolisme de la nourriture se trouve à son apogée.

8 Le sang est placé au cœur du système des représentations de la consommation de viande. Sur le plan symbolique une question se pose : qui va assumer l'acte de tuer, la mort de l'animal ensanglanté provoquant un malaise qu'on ne rencontre pas dans les signifiés qui accompagnent d'autres plats, comme le poisson par exemple. L'angoisse de l'animal qui est sacrifié sur l'autel de la jouissance humaine provoque un sentiment de culpabilité et rappelle l'implacable loi de la nature où le vivant tue le vivant, soulignant ainsi le fait que l'être humain est périssable comme sa nourriture.

Les poètes abordent le symbolisme de la chair sacrifiée dans le cadre de l'évolution historique du genre humain, recherchant le premier coupable dans l'histoire de Caïn et d'Abel, ce qui sous-entend que la cause du premier homicide qui contamina dès lors l'être humain par sa quête de sang, était la préférence de Dieu pour le sacrifice de l'animal. C'est ainsi que, sans pour autant le nommer, Varveris accuse ouvertement Dieu d'incitation au meurtre 


\begin{tabular}{|c|c|}
\hline Ev $\propto \rho \chi \underline{n}$ & Au commencement* \\
\hline 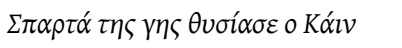 & Caïn fit offrande des produits de la terre \\
\hline 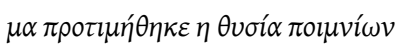 & mais le sacrifice des troupeaux d'Abel \\
\hline$\tau O v^{\prime} A \beta \varepsilon \lambda$ & lui fut préféré. \\
\hline 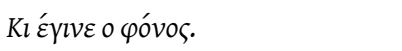 & Et le sacrifice eut lieu. \\
\hline 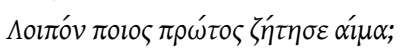 & Alors, qui le premier, a exigé le sang? \\
\hline
\end{tabular}

*Varveris Yannis, l'Homme seul (2009), Poèmes 2001-2013, p. 95

10 Vassilis Ladas associe, lui aussi, symboliquement le premier homicide à la consommation de viande et à l'évolution de notre culture fratricide, rejetant ainsi la responsabilité sur Dieu et sur son choix de n'accepter que l'offre d'Abel.

\begin{tabular}{|c|c|}
\hline 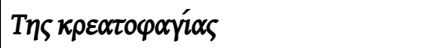 & De la carnivorité* \\
\hline 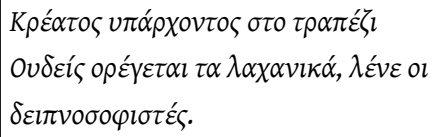 & $\begin{array}{l}\text { Lorsqu'il y a de la viande sur la table } \\
\text { Personne n'a d'appétit pour les légumes, } \\
\text { disent les Deipnosophistes. }\end{array}$ \\
\hline 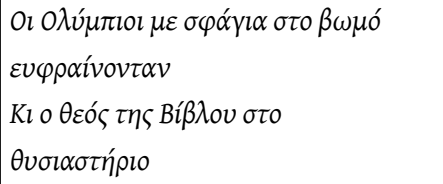 & $\begin{array}{l}\text { Les dépouilles sur l'autel } \\
\text { enchantaient les Dieux de l'olympe } \\
\text { Et le Dieu de la Bible sur l'autel } \\
\text { sacrificiel }\end{array}$ \\
\hline 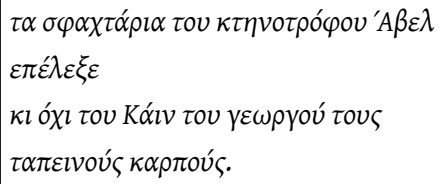 & $\begin{array}{l}\text { Choisit l'animal abattu d'Abel le } \\
\text { pasteur } \\
\text { Et non du cultivateur Caïn les } \\
\text { humbles produits }\end{array}$ \\
\hline 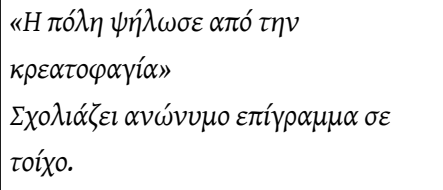 & $\begin{array}{l}\text { "C'est la consommation de viande } \\
\text { agrandi la ville " } \\
\text { Commente une épigramme anonyme } \\
\text { sur un mur. }\end{array}$ \\
\hline 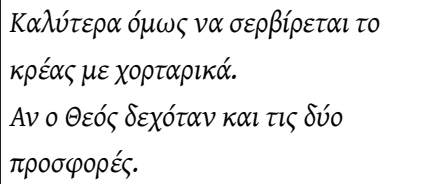 & $\begin{array}{l}\text { Il vaudrait pourtant mieux servir la } \\
\text { viande accompagnée de légumes. } \\
\text { Si Dieu avait accepté les deux } \\
\text { offrandes. }\end{array}$ \\
\hline 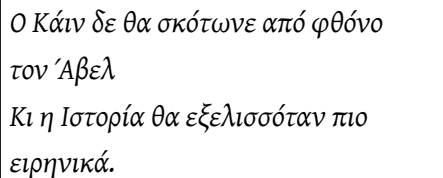 & $\begin{array}{l}\text { Caïn n'aurait pas tué Abel par } \\
\text { jalousie } \\
\text { Et l'Histoire aurait évolué plus } \\
\text { paisiblement. }\end{array}$ \\
\hline
\end{tabular}

*LADAS Vassilis, Dîners, p. 16.

11 Dans son poème la Décoration du repas, Athina Papadaki, elle aussi, pose de manière à la fois autocritique et culpabilisée la question de la consommation de viande en tant que meurtre maquillé, qui touche aux limites du cannibalisme, mais un meurtre raffiné et paré de tout le nécessaire de l'art de la table?.

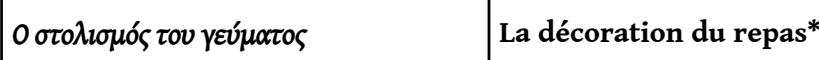




\begin{tabular}{|c|c|}
\hline 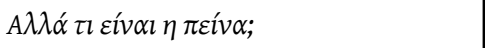 & Mais qu'est-ce que la faim? \\
\hline 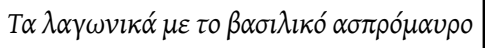 & Bondissants, les limiers au \\
\hline 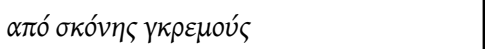 & majestueux noir et blanc \\
\hline$\varepsilon \rho \chi \operatorname{\mu }^{\prime} \varepsilon v \alpha \kappa \alpha \iota \mu \varepsilon \alpha^{\prime} \lambda \mu \alpha \tau \alpha$ & Surgissant d'abîmes de poussière \\
\hline 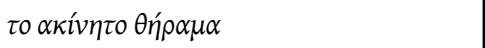 & Maculent soudain, en un frisson de \\
\hline 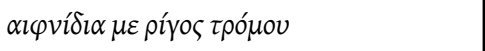 & peur \\
\hline 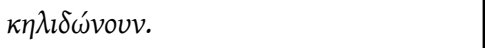 & La proie pétrifiée. \\
\hline 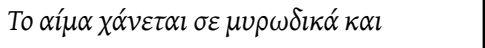 & Le sang se perd dans les aromates et \\
\hline$\alpha \tau \mu o u ́ \varsigma$, & les vapeurs \\
\hline$\sigma \tau \eta \lambda \alpha \mu \pi \varepsilon \rho \eta^{\prime} \tau \rho \alpha \pi \varepsilon \zeta \alpha \rho i \alpha$ & dans la salle à manger flamboyante \\
\hline$\mu \varepsilon \pi \circ \rho \sigma \varepsilon \lambda \alpha^{\prime} v \eta \varsigma \sigma v \mu \pi \varepsilon \rho l \varphi \circ \rho \alpha \dot{\alpha}$ & aux comportements de porcelaine \\
\hline 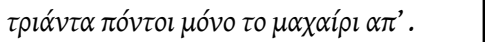 & à trente centimètres de distance \\
\hline$\tau o \pi l \rho o u ́ v l$. & seulement le couteau de la fourchette. \\
\hline 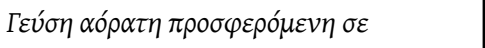 & Saveur invisible servie dans une \\
\hline 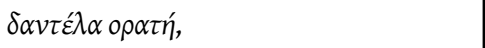 & dentelle visible \\
\hline 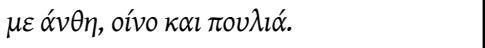 & Avec des fleurs, du vin et des oiseaux. \\
\hline 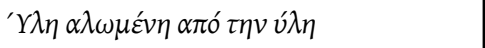 & Matière dominée par la matière \\
\hline 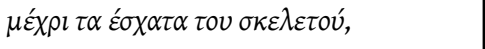 & Jusqu'aux confins du squelette \\
\hline 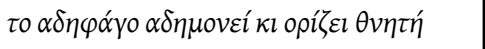 & L'insatiable s'impatiente et qualifie \\
\hline$\tau \eta \zeta \omega \eta \dot{\mu} \mu \alpha$. & notre vie de mortelle. \\
\hline
\end{tabular}

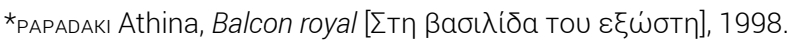

12 Le repas de chair et de sang des hommes "est maculé en un frisson de peur ", avant d'être livré cru sur l'autel de la cuisine. Là où l'odeur persistante du sang sera cachée par les «aromates et les vapeurs » de façon à présenter la viande métamorphosée, purifiée par le meurtre prémédité et la peur de la mort. « La matière est dominée par la matière " et se transforme en une "saveur invisible » et immatérielle, en une nature morte quasiment restituée sur le plan plastique par la poétesse comme les natures mortes ou les «vies immobiles» (still life) des peintres hollandais ${ }^{10}$. Un produit nouveau, dépossédé de ses qualités initiales, décoré d'éléments de la demeure civilisée. Le cru se fait cuit, de physique la condition devient sociale, et le cuisinier est l'intermédiaire entre le monde physique et la consommation humaine, comme LéviStrauss le décrit dans son essai le Cru et le Cuit ${ }^{11}$. Le tout dans un contexte d'avidité, de suralimentation, d'hypocrisie et de futilité.

Dans la poésie de Papadaki, la cuisinière endosse la responsabilité de faire disparaître les traces du sacrifice et de purifier le meurtre, tout en étant elle-même condamnée à contempler la vérité jusqu'à l'os, "jusqu'aux confins du squelette » en assimilant sa 
propre dimension physique à la matière cuisinée qu'elle prépare pour son repas mondain.

\begin{tabular}{|c|c|}
\hline$\Delta \varepsilon \xi \iota \omega ́ \sigma \varepsilon l \varsigma$ & Réceptions* \\
\hline 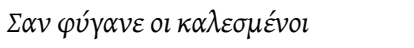 & Quand les invités furent partis \\
\hline 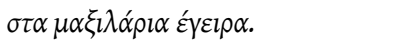 & Je m'allongeai sur les coussins. \\
\hline Koí $\tau \alpha \xi \alpha$. & J'observai. \\
\hline 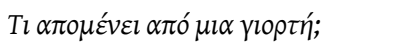 & Que reste-t-il d'une fête? \\
\hline 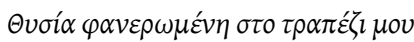 & Sur ma table, un sacrifice avéré \\
\hline 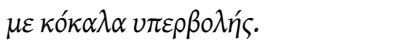 & Et les carcasses de l'outrance. \\
\hline
\end{tabular}

*PAPADAKı Athina, op. cit.

14 L'impact de la pensée de Schopenhauer sur la Volonté affamée devient aussi manifeste dans la poésie de Papadaki: c'est le destin de toute chose dans la nature d'être finalement mangée par quelque élément destructeur ; même celui de l'être humain qui considère pourtant que la nature a été créée pour son utilité personnelle. Schopenhauer rappelle les effets de la nature humaine insatiable. Homo homini lupus, «l'homme est un loup pour l'homme $»^{12}$. Chez Papadaki, la consommation de viande ne semble pas très éloignée de l'anthropophagie ${ }^{13}$.

\section{« De la mort fraîcheurs... »}

La thématique de la nourriture suppliciée et de son assimilation au destin humain ne concerne cependant pas seulement les repas carnivores. Chez Varveris, l'allégorie de la nourriture culpabilisante s'étend également de façon inattendue aux fruits de mer. Ici le sang n'intervient pas en tant qu'alibi - d'accaparement de la force animale - et ne souille pas la conscience de l'assiette civilisée; aucun besoin donc de traiter et de transformer l'aliment afin qu'il puisse être consommé sans culpabilité. Cependant, chez Varveris, les crustacés et les poissons acquièrent un symbolisme tout particulier, plus fort que celui qui accompagne la viande. La denrée vivante n'est pas similaire à la denrée cuisinée ni même à celle qui est crue. Dans les poèmes suivants, la denrée ne «tressaute " pas seulement; elle agonise. Ce qui, pour l'homme, constitue un critère de qualité, se trouve être, au même instant, un instant tragique pour un autre être, aussi vivant que lui.

\begin{tabular}{|c|c|}
\hline 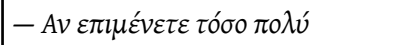 & - Si vous insistez tant \\
\hline 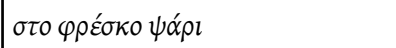 & pour avoir du poisson frais \\
\hline 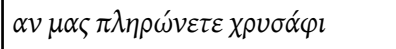 & si vous nous achetez à un prix si exorbitant \\
\hline 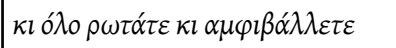 & et toujours demandez et doutez tant \\
\hline 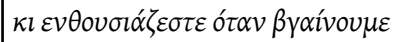 & et si nous voir sortir de l'eau, \\
\hline 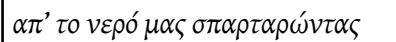 & agonisants, vous exalte tant \\
\hline
\end{tabular}




\begin{tabular}{|c|c|}
\hline 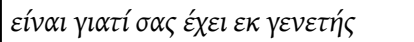 & c'est parce que dès votre naissance \\
\hline$\sigma \varphi \rho \alpha \gamma i ́ \sigma \varepsilon l$ & la saveur du sel de la mort \\
\hline 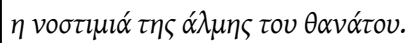 & vous a marqués.* \\
\hline
\end{tabular}

* varveris Yannis, Poèmes 2001-2013, p. 293.

\begin{tabular}{|c|c|}
\hline 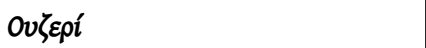 & Ouzeries* \\
\hline 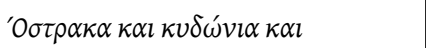 & Coquillages, pétoncles et palourdes \\
\hline$\gamma v \alpha \lambda_{\imath} \sigma \tau \varepsilon \rho \varepsilon ́ \varsigma$ & à l'agonie \\
\hline 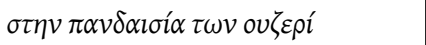 & dans une débauche \\
\hline$\chi \alpha \rho \circ \pi \alpha \lambda \varepsilon v ́ o v \tau \alpha \varsigma$ & d'ouzeries, \\
\hline 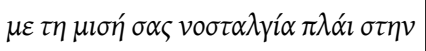 & la moitié de votre nostalgie gisant \\
\hline$\alpha \alpha \lambda \eta$ & tout contre l'autre \\
\hline$\tau o v \tau \varepsilon \lambda \varepsilon v \tau \alpha i_{0} \sigma \pi \alpha \sigma \mu o ́ \sigma \alpha \varsigma \tau \sigma \nu$ & vous dansez votre dernier spasme \\
\hline 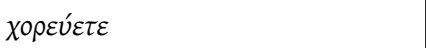 & comme le grand sanglot qui monte en \\
\hline 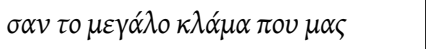 & qui monte en \\
\hline$\alpha v \varepsilon \beta \alpha i ́ v \varepsilon l$ & nous \\
\hline 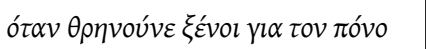 & quand se lamentent des inconnus \\
\hline$\mu \alpha \varsigma$ & sur nos peines ; \\
\hline$o ́ \mu \omega \varsigma, \kappa v \delta \omega^{\prime} v l \alpha \mu o v$ & et pourtant, mes pétoncles, \\
\hline$\gamma \imath \alpha \tau \alpha \delta i k \alpha \dot{\alpha} \tau o v \varsigma \kappa \lambda \alpha i ́ \gamma \alpha \nu$ ol & les hommes pleuraient \\
\hline$\alpha^{\alpha} v \theta \rho \omega \pi 0 l$ & sur eux-mêmes \\
\hline$\alpha \pi \alpha ́ \tau \eta o^{\prime} \lambda \alpha \tau \alpha \delta \alpha^{\prime} \kappa \rho v \alpha$ & duperie que toutes ces larmes, \\
\hline 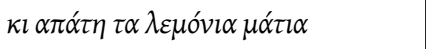 & imposture ces citrons, ces yeux \\
\hline$\pi \circ v \theta \alpha \delta \alpha \kappa \rho v ́ \sigma o v v \pi \alpha^{\prime} v \omega \sigma \alpha \varsigma \gamma l \alpha \nu \alpha$ & qui larmoieront sur vous \\
\hline 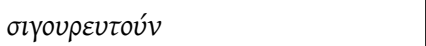 & pour voir \\
\hline 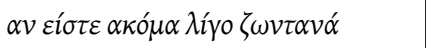 & si vous êtes encore un peu vivants \\
\hline 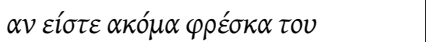 & si vous êtes encore de la mort \\
\hline$\theta \alpha v \alpha ́ \tau o v$. & fraîcheurs. \\
\hline
\end{tabular}

*VARVERIS Yannis, Poèmes 2001-2013, p. 293.

16 La singularité de ces poèmes consiste dans le fait que l'homme ne s'approprie pas les qualités symboliques de l'aliment mais au contraire, l'organisme devant être mangé est personnifié et acquiert des qualités humaines: il parle, ressent de la nostalgie, philosophe, danse, agonise. Varveris donne voix et sentiments aux aliments leur conférant ainsi des éléments anthropomorphiques. La communication intellectuelle et orale entre aliments et consommateur souligne qu'il ne s'agit plus d'un " meurtre » qui s'est déroulé de façon impersonnelle et anonyme, avant que le repas soit servi, mais 
d'un affrontement inégal, à vif, d'un "face à face", au cours duquel le plaisir du mangeur se lie à l'angoisse d'un autre organisme vivant, doué, en outre, de parole. L'aponie et l'ataraxie du bourreau sont obtenus au prix de la mort dans l'angoisse de sa victime et la sensibilité varverique concorde avec la position bien connue de Marguerite Yourcenar " il me déplaît de digérer les agonies " ${ }^{14}$. La fraîcheur convoitée, le poisson qui tressaute, les crustacés qui « dansent leur dernier spasme », tout souligne à l'extrême le sadisme humain et l'imposture de la prétendue compassion du plus fort, qui se penche sur son repas « de la mort fraîcheurs ", la larme à l'œil, prêt à le dévorer vivant. La "saveur de la mort » dépasse l'alibi de la loi naturelle de survie qu'évoque la phrase « ta mort est ma vie ». La nourriture qui a un visage, selon la notion morale que Lévinas attribue au visage, invoque notre respect et nous interdit de tuer ${ }^{15}$. C'est à ce genre d'éthique que se range Varveris quand il dénonce ce que nous résumerons comme étant « ta mort en martyre est ma jouissance ».

\section{La suralimentation de l'individualisme}

Mais si dans la poésie contemporaine domine la culpabilité face à la nourriture, sur le plan symbolique, la dimension sociale qui est attribuée à la signification du repas à l'époque de la crise humanitaire dont on parle tant, est aussi importante.

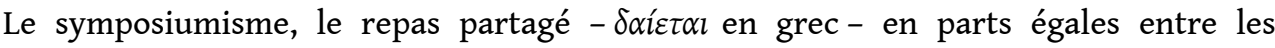
convives, de façon à ce que tous partagent la même quantité et la même saveur, disparaît. La réciprocité a laissé place à l'individuel. Cependant la nutrition est l'acte social par excellence de partage-communion et la composition du con-vivium est presqu'aussi importante que la composition du plat ${ }^{16}$. Le corps social est un macrocosme du corps physique et selon l'anthropologue Mary Douglas,

exactement comme le corps social hausse des murs, surveille ses portes, interdit

l'entrée [...], le corps physique prend soin de ses limites, de ses entrées et de ses sorties comme la bouche ${ }^{17}$.

Dans ce contexte, nous repérons des poèmes qui ont pour but de rappeler le besoin de survie des exclus du corps social et de la table sociale, de ceux qui ont été rejetés comme étant inutiles et qui se nourrissent des déchets que nous expulsons de notre table et de notre corps : tout ce que nous jetons aux ordures, tout ce qui est pourri et périmé, nos restes.

20 Tassoula Karageorgiou se demande succinctement ${ }^{18}$ :

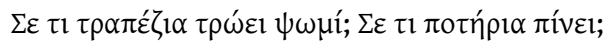

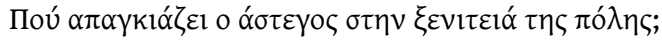

Sur quelles tables mange-t-il du pain? Dans quels verres boit-il ?

Où se réfugie le sans-abri, banni dans la ville?

21 Dans son recueil qui a pour titre Dîners, Vassilis Ladas parle du partage des denrées, de la cohésion sociale et de la solidarité entre les convives ${ }^{19}$ :

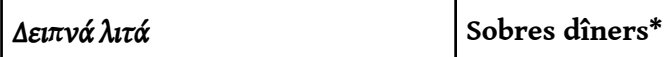




\begin{tabular}{|c|c|}
\hline 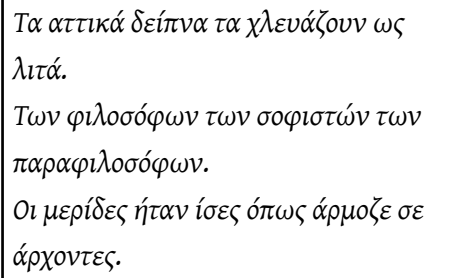 & $\begin{array}{l}\text { Les dîners attiques sont raillés } \\
\text { à cause de leur frugalité. } \\
\text { Ces dîners des philosophes, des } \\
\text { sophistes et des prétendus philosophes. } \\
\text { Les parts étaient égales comme il se } \\
\text { doit à des seigneurs. }\end{array}$ \\
\hline 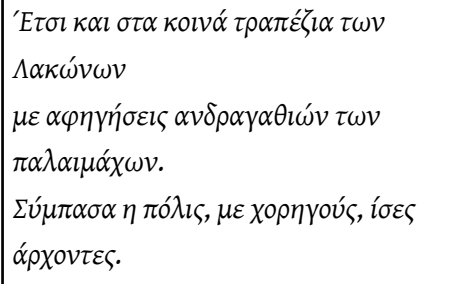 & $\begin{array}{l}\text { Mais à la table ordinaire des } \\
\text { Lacons aussi } \\
\text { Là où l'on relatait les prouesses des } \\
\text { vétérans } \\
\text { Grâce aux bienfaiteurs, la ville toute } \\
\text { entière avait des parts égales. }\end{array}$ \\
\hline 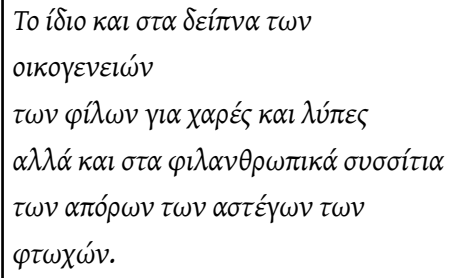 & $\begin{array}{l}\text { Pareillement dans les dîners } \\
\text { familiaux, } \\
\text { Dans ceux donnés par les amis } \\
\text { à l'occasion de joies et de peines } \\
\text { Et dans les soupes populaires } \\
\text { Pour les indigents, les sans-abri, les miséreux }\end{array}$ \\
\hline IбEs $\mu \varepsilon \rho i ́ \delta \varepsilon \varsigma \kappa \alpha \tau \alpha \rho \chi \eta ́ v ~ \tau o ~ \varphi \alpha \gamma \eta \tau o ́$. & En parts égales avant tout, la nourriture. \\
\hline 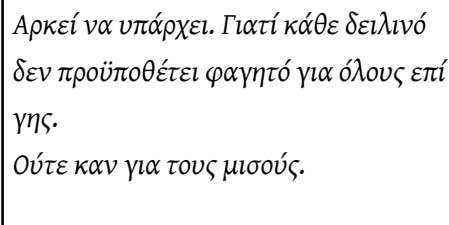 & $\begin{array}{l}\text { S'il y en a en suffisance. Car chaque } \\
\text { jour ne signifie pas forcément à } \\
\text { manger pour tous sur cette terre. } \\
\text { Même pas pour la moitié d'entr } \\
\text { nous. }\end{array}$ \\
\hline
\end{tabular}

*LADAS Vassilis, Dîners, 2011, p. 14.

Dans son poème intitulé « Des empoisonnés » et qui fait partie du même recueil, Ladas prend prétexte d'un slogan qu'il a vu sur une poubelle et qu'il reprend dans les deux derniers vers. Considérons le mot « involontaires » et les responsabilités qu'il confère :

\begin{tabular}{|c|c|}
\hline$T \omega \nu \delta \eta \lambda \eta \tau \eta \rho 1 \alpha \sigma \mu \varepsilon ́ v \omega \nu$ & Des empoisonnés* \\
\hline 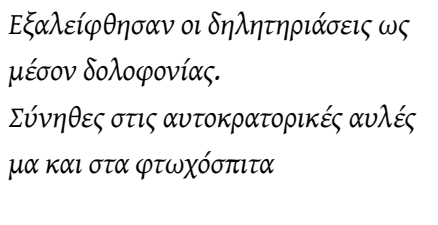 & $\begin{array}{l}\text { Les empoisonnements comme } \\
\text { instruments homicidaires ont } \\
\text { disparu. } \\
\text { Courants dans les cours impériales } \\
\text { Mais chez les pauvres aussi }\end{array}$ \\
\hline 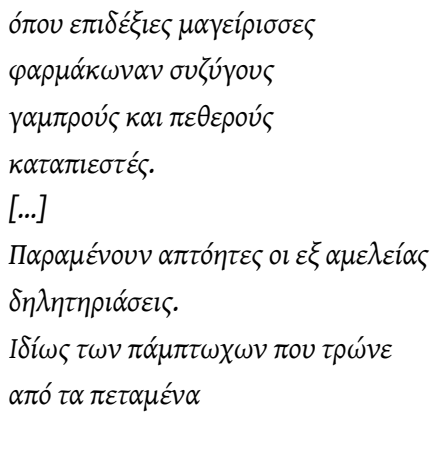 & $\begin{array}{l}\text { où d'habiles cuisinières } \\
\text { empoisonnaient leurs époux, } \\
\text { leurs beaux-frères et beaux-pères } \\
\text { tyranniques } \\
\text { [...] } \\
\text { Les empoisonnements } \\
\text { involontaires } \\
\text { persistent. } \\
\text { Ceux des miséreux surtout } \\
\text { qui mangent }\end{array}$ \\
\hline
\end{tabular}




\begin{tabular}{|c|c|}
\hline 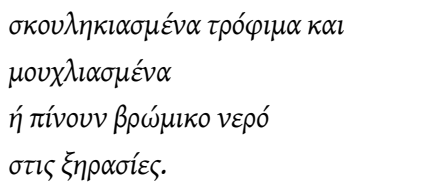 & $\begin{array}{l}\text { les denrées véreuses et moisies qu'on } \\
\text { a jetées } \\
\text { ou boivent de l'eau sale } \\
\text { dans les flaques }\end{array}$ \\
\hline 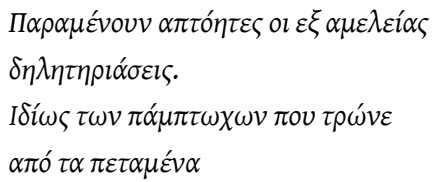 & $\begin{array}{l}\text { Même si leur sang acquiert des } \\
\text { anti-corps } \\
\text { grâce à chaque petite dose } \\
\text { quotidienne de poison. }\end{array}$ \\
\hline 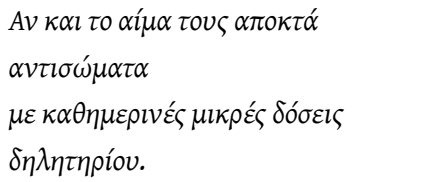 & $\begin{array}{l}\text { Même si leur sang acquiert des } \\
\text { anti-corps } \\
\text { grâce à chaque petite dose } \\
\text { quotidienne de poison. }\end{array}$ \\
\hline 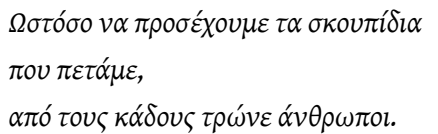 & $\begin{array}{l}\text { Attention donc aux ordures } \\
\text { que nous jetons, } \\
\text { dans les poubelles mangent des êtres humains. }\end{array}$ \\
\hline
\end{tabular}

*Ladas Vassilis, Dîners, 2011, p. 17.

\section{En conclusion}

La faim en tant que rappel du besoin de survie, l'appétit et la saveur en tant que désir de dépassement de la seule survie, la « morsure sur les choses $»^{20}$ en tant que poursuite $\mathrm{du}$ bonheur, sont inscrits dans les poèmes plus comme un manque, que comme un achèvement. Au-delà des intemporelles sources existentielles ou, peut-être même avec elles, émerge dans la poésie de nos jours - au niveau mondial - une problématique contemporaine d'une actualité exceptionnelle et qui concerne une dimension éthique très différente du principe épicurien du bonheur que nous avons souligné dès le début de notre texte, problématique que nous n'avons cependant remarquée dans les poèmes qu'en tant qu'absence. Il s'agit là d'un genre d'éthique et d'alerte existentielle suggérée dans la poésie de Varveris ou celle de Papadaki et qui se résume dans l'opinion de Yourcenar relative à un système de valeurs qui respectera tout à la fois l'angoisse de l'être humain pour la survie et l'angoisse de l'animal élevé et abattu pour être consommé ${ }^{21}$. Et bien sûr, on ne peut qu'associer la discussion sur les «droits des animaux» à la critique sur la goinfrerie et la suralimentation de l'homme contemporain dans les sociétés développées et le contraste tragique entre la sousalimentation et la lutte pour la survie du prochain non privilégié, comme Ladas le souligne avec force dans sa poésie ${ }^{22}$.

Les repas culpabilisés de la poésie contemporaine parlent de l'angoisse et non du plaisir. Ils assimilent le destin mortel de la nourriture vivante au caractère temporaire et périssable de la condition humaine et témoignent de l'« anorexie de l'existence $»^{23}$. 


\section{BIBLIOGRAPHIE}

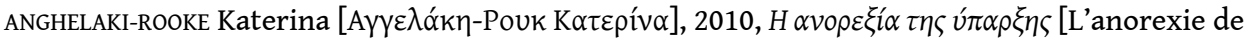
l'existence], Athènes : Éditions Kastaniotis.

DOUGLAS Mary, 1975, Implicit meanings, London: Routledge and Keagan Paul.

DOUGLAS Mary, 1966, Purity and danger: An analysis of the concepts of pollution and taboo, London:

Routledge and Keagan Paul.

GOODY Jack, 1982, Cooking, cuisine and class: A study in Comparative Sociology, Cambridge: Cambridge University Press.

JAQUET Chantal, 2001, le Corps, Paris : PUF.

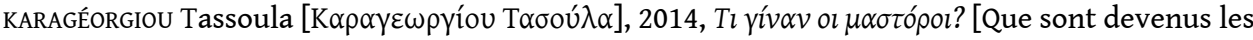
artisans ?], Athènes : Éditions Gavrielidès.

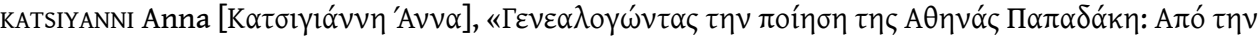

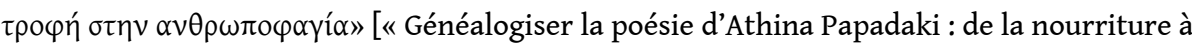
l'anthropophagie »], <duendemagazine.gr>.

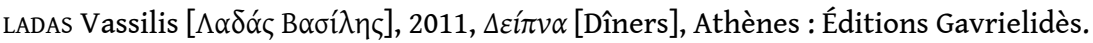

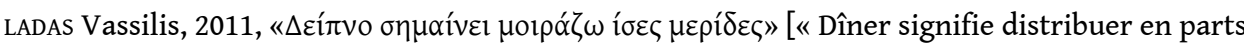

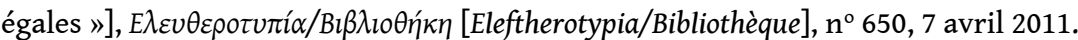

LÉVI-STRAuss Claude, 1964, Mythologiques : le cru et le cuit, Paris : Plon.

LÉVINAS Emmanuel, 1982, Éthique et infini, Paris : Fayard.

LÉVINAS Emmanuel, 1971/1990, Totalité et infini : essai sur l'extériorité, La Haye : Nijhoff, Paris : Le Livre de Poche/Biblio essais.

MÉCHIN Colette, 1997, « la Symbolique de la viande », in PAILLAT Monique (dir.), le Mangeur et l'Animal : mutations de l'élevage et de la consommation, Paris : Autrement, Coll. Mutations/Mangeurs, $\mathrm{n}^{\circ} 172$, p. $121-134$.

MENNEN Stephen, 1987, Français et Anglais à table, Paris : Flammarion.

MUCHNIK José, 2010, « Nourriture et poésie », $250^{\mathrm{e}}$ anniversaire de l'Académie d'Agriculture de France, Séance Culture et Gastronomie, 24 novembre 2010, http://www.academie-agriculture.fr, consulté le 10 décembre 2015.

OWEN SCHAEFER G. \& SAVULESKU Julian, 2014, "The ethics of producing in vitro meat", Journal of Applied Philosophy, v. 31, no 2, pp. 188-202.

PAILlat Monique (dir.), 1997, le Mangeur et l'Animal : mutations de l'élevage et de la consommation, Paris : Autrement, Coll. Mutations/Mangeurs, $\mathrm{n}^{\circ} 172$.

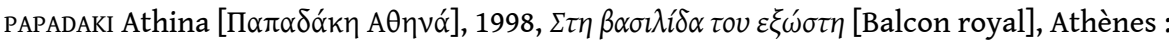
Éditions Kastaniotis.

REGAN Tom, 2004, The Case for Animal Rights, Berkeley: University of California Press.

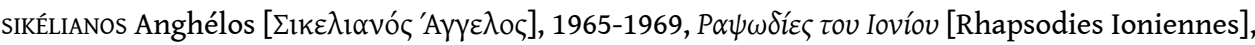

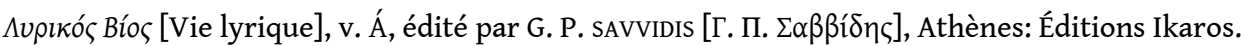


SINGER Peter, 1975, Animal Liberation, New York: Avon Books.

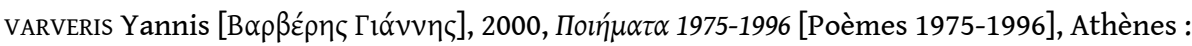

Éditions Kédros.

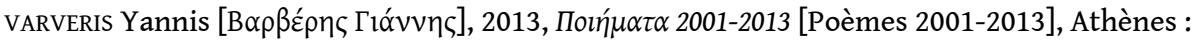

Éditions Kédros.

YOURCENAR Marguerite, 1968, l'Euvre au noir, Paris : Gallimard.

YOURCENAR Marguerite, 1980, les Yeux ouverts : entretiens avec Matthieu Galey, Paris : Éditions du Centurion.

\section{NOTES}

1. Le Larousse définit l'ataraxie comme " quiétude absolue de l'âme, idéal du sage, selon l'épicurisme et le stoïcisme ". Le dictionnaire CNRTL définit le terme philosophique d'ataraxie comme "tranquillité, impassibilité d'une âme devenue maitresse d'ellemême au prix de la sagesse acquise soit par la modération dans la recherche des plaisirs (Épicurisme)».

2. Voir JAQUET Chantal, le Corps, p. 268-274.

3. In Lettre à Ménécée, 128-129 et Sentences vaticanes, 33.

4. LÉVINAS Emmanuel, le Temps et l'Autre, p. 45.

5. SIKÉLIANOS Angelos, Rhapsodies Ioniennes, 1909, Vie lyrique, v. A'.

6. Voir DOUGLAS Mary, "Deciphering a meal" in Implicit meanings, pp. 249-275.

7. VARVERIS Yannis, le Miracle annulé (1996), Poèmes 1975-1996, p. 397.

8. MÉCHIN Colette, "La symbolique de la viande " in Paillat Monique, le Mangeur et l'Animal. Mutations de l'élevage et de la consommation, p. 121-134: «Les abats posent un autre problème, celui de l'identification des morceaux : un cœur, une cervelle, voire un estomac farci, signalent dès le premier coup d'œil leur origine. Or notre époque, en s'éloignant de ses origines paysannes, répugne à ce rappel de la réalité. », p. 127.

9. «En masquant par d'ingénieux décors ou de savants procédés de cuisson l'aspect cruel des viandes dépecées, l'art de la cuisine contribue certainement à l'adoucissement des mœurs ", écrit au XIX ${ }^{e}$ siècle Châtillon-Plessis cité par MENNEN Stephen, Français et Anglais à table, 1987, p. 442-443.

10. Pour la critique faite par Schopenhauer dans les «natures mortes » des peintres de l'école hollandaise qui concerne l'appétit, voir JACQUETTE Dale, The philosophie of Schopenhauer, 171-175.

11. LÉVI-STRAUSS Claude, Mythologiques. Le cru et le cuit, 1964. Voir également Goody Jack, Cooking, cuisine and class, A study in Comparative Sociology, 1982.

12. SCHOPENHAUER Arthur, Die Welt als Wille und Vorstellung, I: 147. Comme le souligne aussi Schopenhauer, la phrase appartient à Plaute: "Lupus esthomohomini" (PLAUTE, Asinaria, 495). Voir JACQUETTE Dale, The philosophy of Schopenhauer, p. 108-114.

13. Voir également KATSIYANNI Anna, «Généalogiser la poésie d'Athina Papadaki : De la nourriture à l'anthropophagie ». 
14. YOURCENAR Marguerite, les Yeux ouverts. Entretiens avec Matthieu Galey, 1980, p. 287-302: «Mais je n'oublie surtout pas l'agonie du poisson tiré par la ligne ou tressautant sur le pont d'une barque. Tout comme Zénon, il me déplaît de digérer les agonies ». Voir également YOURCENAR Marguerite, l'Euvre au noir, 1968, p. 176.

15. LÉVINAS Emmanuel, "Visage et éthique » in Totalité et infini, p. 215-220 et Éthique et infini, p. 106: "La relation au visage est d’emblée éthique. Le visage est ce qu'on ne peut tuer, ou du moins ce dont le sens consiste à dire "Tu ne tueras point" ».

16. Voir MUCHNIK José, « Nourriture et poésie », p. 6-7.

17. Douglas Mary, Purity and danger. An analysis of the concepts of pollution and taboo, pp. 115.

18. KARAGÉORgIOU Tassoula, Que sont devenus les artisans, p. 11.

19. Dans une des interviews qu'il a données, Ladas soutient «l'activisme des mots » et commente son recueil de la façon suivante : «Comment puis-je autrement faire face au bombardement d'émissions télévisées sur les recettes gourmets, à une époque où le caractère sacré du repas a disparu et où les morts de faim et de soif se multiplient ? ", in « Dîner signifie partager en parts égales » (interview), 2011.

20. LÉVINAS Emmanuel, Totalité et infini: «Cette morsure sur les choses que, par excellence, comporte l'acte de manger [...]», p. 135.

21. Il existe de nos jours, d'ailleurs, un grand débat sur la production in vitro des produits "éthiques » de la viande ; ses partisans parlent de résolution des problèmes éthiques et environnementaux que posent l'abattage des animaux et de la réduction du prix de revient qui contribuera au règlement de la crise humanitaire. Consulter également owen SCHAEFER G. and SAVULESKU Julian, "The ethics of producing in vitro meat”, 2014 ;SINGER Peter, Animal Liberation, 1975 ; REGAN Tom, The Case for Animal Rights, 2004.

22. Voir LADAS Vassilis, op. cit. : «Derrière les lignes bien sûr, je cherche à esquisser les traces d'un jeune garçon, un immigré qui a faim ou qui cherche de l'eau, tout comme la solitude du malade qui mange seul [...] Et je n'oublie pas un chat noir qui, à la veille des élections de 2007, s'était précipité sur la route, attiré par l'odeur de nourriture qui lui semblait venir du trottoir d'en face et que les voitures écrasèrent dans le tumulte. ", in « Dîner signifie distribuer en parts égales ", op. cit.

23. Nous empruntons le titre au recueil de Katerina ANGHELAKI-ROOKE, l'Anorexie de l'existence, 2010. Le poème homonyme du début du recueil commence par les vers suivants : «Je n'ai pas faim, je n'ai pas mal, je ne pue pas/peut-être que je souffre tout au fond de moi sans le savoir/je fais semblant de rire/je ne désire pas l'impossible/ni le possible/ [...]». 


\section{RÉSUMÉS}

La présente étude vise à examiner la dimension symbolique de l'alimentation dans la poésie grecque contemporaine. La nourriture nous rappelle notre condition corporelle et les limites de la substance matérielle. Les poètes mettent en relation la nourriture et la mort tout en soulignant la valeur de la convivialité dans les années de la crise économique.

The present study aims to detect the symbolic dimension of food in contemporary Greek poetry. Nourishment reminds us of our corporeal condition and defines the limits of material substance. The poets relate food to death by underlining the culpability of existence. They also reveal the value of sharing in the years of crisis and individualism.

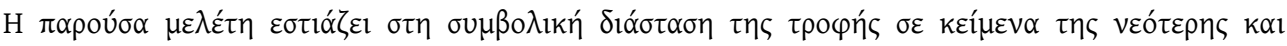

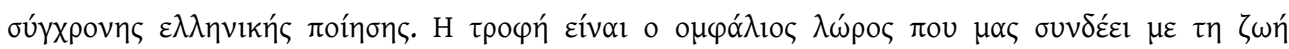

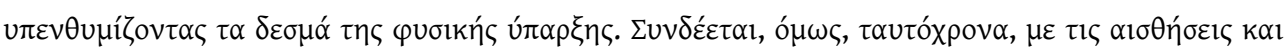
$\tau \eta v$ a

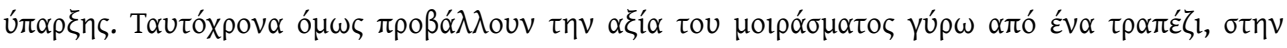

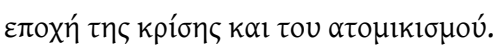

\section{INDEX}

Index géographique : Grèce

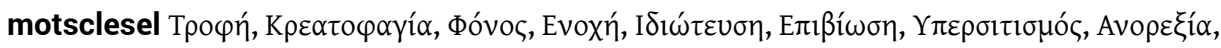

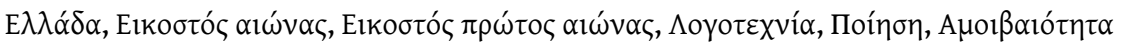

Keywords : Food, Carnivority, Murder, Guilt, Individualism, Survival, Over-nourishment, Anorexia, Greece, Twentieth Century, Twenty First century, Literature, Poetry

Mots-clés : nourriture, nourriture, carnivorité, carnivorité, meurtre, meurtre, culpabilité, culpabilité, individualisme, individualisme, survie, survie, suralimentation, suralimentation, anorexie, Grèce, Grèce, 20e siècle, 20e siècle, 21e siècle, 21e siècle, littérature, littérature, poésie, poésie, réciprocité, réciprocité

Thèmes : Littérature, Poésie

motsclestr Yiyecek, Etobur, Cinayet, Suçluluk, Bireycilik, Survival, Aşırı yeme, Anoreksiya, Yunanistan, Yirminci yüzyıl, Yirmi birinci yüzyıl, Edebiyat, şiir

motsclesmk ХРАНА, МЕСОЈАДИ, УБИСТВО, ВИНА, ИНДИВИДУАЛИЗАМ, ПРЕЖИВУВАҢЕ, ПРЕЈАДУВАњЕ, АНОРЕКСИЈА, ГРЦИЈА, ДВАЕСЕТТИОТ ВЕК, ДВАЕСЕТ И ПРВИОТ ВЕК, ЛИТЕРАТУРА,

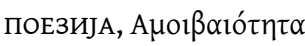

Index chronologique : vingtième siècle, vingt-et-unième siècle

\section{AUTEUR}

\section{THÉODOULI ALEXIADOU}

Enseignante, Université d'Athènes 